\title{
Serum ischemia-modified albumin level returns to its premenopausal level with 1-year hormone therapy in healthy menopausal women
}

\author{
Esra Can Cetin ${ }^{1}$, Suleyman Guven ${ }^{1}$, Hidayet Sal ${ }^{1}$, Emine Seda Guvendag Guven ${ }^{1}$, Ahmet Mentese ${ }^{2}$ \\ ${ }^{1}$ Karadeniz Technical University School of Medicine, Department of Obstetrics and Gynecology, Trabzon, Turkey \\ 2Program of Medical Laboratory Techniques, Vocational School of Health Sciences, Karadeniz Technical University, Trabzon, Turkey
}

\begin{abstract}
Introduction: To assess the effect of hormone therapy (HT) on serum ischemia modified albumin (IMA) levels in healthy menopausal women.

Material and methods: Thirty surgical menopausal women who were admitted to our menopausal polyclinic during a 1-year period and diagnosed with menopause and planned to have HT for menopausal symptoms were enrolled in this prospective study. The serum iMA levels were recorded before and after ( 3 months, 6 months, 12 months later) hormone treatment ( 2 mg estradiol hemihydrate).

Results: The mean age of women was $47.60 \pm 2.34$ years. The mean serum IMA levels were $0.610 \pm 0.096$ absorbance units (ABSU) at the beginning and $0.484 \pm 0.080 \mathrm{ABSU}$ after 3 months of hormone therapy. Following 6 months of hormone therapy, serum IMA level was $0.546 \pm 0.075$, and reached $0.580 \pm 0.089$ ABSU following 12 months of therapy.

Conclusions: These findings suggest that HT may not block the menopause induced ischemia process. Although HT had a positive effect on serum IMA levels following 3 months' use, serum IMA levels returned to baseline levels after 12 months' use. Based on this study's findings, long-term use of HT may not have a positive effect on cardiovascular disease protection.
\end{abstract}

Key words: IMA, menopause, hormone therapy, cardiovascular risk.

\section{Introduction}

The protective effect of ovarian activity on diseases of the cardiovascular system in women is known. Accordingly, there is an increased risk of cardiovascular disease (CVD) in menopause. In studies conducted to date, estrogen had positive effects on the cardiovascular system and had a cardioprotective effect. Data in recent years have questioned the notion, which has been believed for many years, that hormone therapy (HT) has a protective effect on the development and progression of coronary artery disease (CAD). Until the release of the Women's Health Initiative (WHI) study, HT was given for menopausal symptoms and treatment was extended until later menopausal periods, believing that it reduced the risk of CVD. This belief arises from the fact that almost all observational studies indicate that HT reduces the risk of CVD and that there has been a lot of evidence available that these treatments have a positive effect on the lipid pattern and maintain vascular functions. As a result of the WHI study, it was suggested that HT should not be started for the primary prevention of CAD and treatment should not be continued in patients who received it before [1].
Hormone therapy in the case of menopause does not provide benefit in terms of eliminating the risks of cardiovascular diseases. However, it provides important benefits in terms of preventing vasomotor symptoms, genitourinary atrophy symptoms and osteoporosis due to menopause. Hormone therapy is also useful in preventing chronic systemic diseases that increase morbidity during menopause [2].

Acute ischemic events increase suddenly in women after menopause. Estrogen deficiency is the main factor responsible for this change. Estrogen reduces cell hypertrophy, increases the elasticity of the vascular wall, and causes less luminal narrowing, although it is likely to be at the same rate as atherosclerosis. With the menopause process, an increase in cholesterol levels is observed. In this process, there is also a tendency to coagulation due to an increase in procoagulant factor VII, fibrinogen and plasminogen activator inhibitor-1 levels in the hemostatic system in the menopause. This explains the increased ischemic conditions after menopause [3, 4].

In our previous study [5], it was found that HT in menopausal patients with obesity as a cardiovascular risk factor decreased serum ischemia-modified albumin 
levels at 3 and 6 months of use. However, in the literature it has not been clear whether this effect occurs in healthy menopause cases and whether this effect continues after one year of use.

This study aimed to investigate the level of ischemia-modified albumin (IMA) in healthy patients with surgical menopause and thus evaluate the reversible effect of HT on the ischemic process.

\section{Material and methods}

Thirty consecutive women, 45-55 years old, who were evaluated for surgical menopause (having a history of total abdominal hysterectomy and bilateral salpingoophorectomy (TAH + BSO) for benign reasons) at the current clinic, and suitable for HT use, were evaluated for this prospective study. Ethic committee approval was obtained from the Research Ethics Committee dated 29.06.2009 and numbered 2009/54.

\section{Case selection}

During the 10-month study period, 190 surgical menopausal women who had undergone TAH + BSO operation in our clinic were evaluated in terms of inclusion criteria. Estradiol hemihydrate (Estrofem film tablet, Novo Nordisk, Istanbul, Turkey) was prescribed to the patients who met the inclusion criteria in the sixth week after TAH + BSO.

Criteria for inclusion in the research:

- accepting consent to participate in the trial and signing the form,

- surgical menopausal women aged 45-55 (having TAH + BSO for benign reasons),

- presence of vasomotor or menopausal symptoms (moderate to severe),

- no systemic disease or infectious disease in the past 2 weeks,

- not taking any other HT or medication until $6^{\text {th }}$ postoperative week,

- no contraindications for HT in routine menopause evaluation,

- willingness to take HT,

- no smoking.

Criteria for exclusion from the study:

- any systemic disease presence (such as diabetes, hypertension, coronary artery disease, hyperlipidemia, endocrine, renal or pulmonary diseases $(n=76)$,

- smoking ( $n=33$, in addition, 16 women had a history of both systemic disease and smoking),

- contraindications for HT $(n=11)$,

- failure to follow-up $(n=18)$,

- inability to complete the three months of HT $(n=14)$,

- quitting HT or other medical reasons during HT $(n=4)$,
- cases reported as preinvasive and invasive genital tumors as a result of TAH $+\mathrm{BSO}(n=3)$,

- cases reported as suspicious findings in the mammography report $(n=2)$.

A blood sample was taken four times during the study, just before starting HT (at 6 weeks after TAH + BSO), at 3 months of HT, at 6 months of HT, and at 12 months of HT. After 8 hours of fasting in each period, 5-7 cc of blood sample was drawn into a heparin-free biochemistry tube around 8.00-9.00 a.m. All blood samples were centrifuged at $900 \mathrm{~g}$ for 10 minutes and collected in the cabinets of the Biochemistry Department at $-80^{\circ} \mathrm{C}$ until all the blood samples collection were completed.

\section{Hormone therapy usage posology}

Estradiol hemihydrate containing preparations (Estrofem film-coated tablets, $2.0 \mathrm{mg}$, Novo Nordisk, Istanbul, Turkey, 12 boxes) were given and 1 tablet per day ( $2 \mathrm{mg} /$ day) was prescribed. Patients were recommended to reapply at the $3^{\text {rd }}, 6^{\text {th }}$, and $12^{\text {th }}$ months of treatment following drug usage.

\section{Measuring serum ischemia-modified albumin level}

After collecting all blood samples, serums were re-dissolved, and serum IMA levels were checked. IMA level was evaluated by the rapid colorimetric method developed by Barr-Or as an absorbance unit [6]. For IMA, the results were recorded in the spectrophotometer at appropriate wavelengths, and the results were recorded as absorbance units (ABSU).

\section{Statistical methods}

All data were entered by coding in the SPSS 10.0 package program. One way analysis of variance (with Bonferroni correction) was performed in the related sample. $P$-values $<0.05$ were considered statistically significant.

\section{Results}

The mean age in the study group was $47.60 \pm 2.34$ years. The general clinical features of the patients are given in Table 1. The most common $\mathrm{TAH}+\mathrm{BSO}$ indication was determined as myoma uteri (46.7\%).

While the mean serum IMA level before HT was $0.610 \pm 0.096 \mathrm{ABSU}$, the mean serum IMA level was $0.484 \pm 0.080 \mathrm{ABSU}$ in the $3^{\text {rd }}$ month, $0.546 \pm 0.075$ ABSU in the $6^{\text {th }}$ month, and $0.580 \pm 0.089$ ABSU in the $12^{\text {th }}$ month after hormone therapy. The change and statistical analysis (one way analysis of variance in related 
Table 1. General clinical features in the research group

\begin{tabular}{|c|c|}
\hline \multicolumn{2}{|l|}{ Clinical features } \\
\hline Age (years) & $47.60 \pm 2.34(45-52)$ \\
\hline Weight (kg) & $74.43 \pm 10.83(51-93)$ \\
\hline BMI $\left(\mathrm{kg} / \mathrm{m}^{2}\right)$ & $26.31 \pm 2.25(22-30)$ \\
\hline Gravida (no.) & $5.03 \pm 2.55(0-10)$ \\
\hline Parity (no.) & $3.87 \pm 1.85(0-8)$ \\
\hline \multicolumn{2}{|l|}{ Indication of TAH + BSO (\%) ${ }^{a}$} \\
\hline Myoma uteri, abnormal uterine bleeding & $14(46.7 \%)$ \\
\hline Dysfunctional uterine bleeding & $10(33.3 \%)$ \\
\hline $\begin{array}{l}\text { Abnormal uterine bleeding, } \\
\text { endometrial hyperplasia }\end{array}$ & $5(16.7 \%)$ \\
\hline Ovarian cyst & $1(3.3 \%)$ \\
\hline \multicolumn{2}{|l|}{ TAH + BSO Pathology Results (\%) ${ }^{a}$} \\
\hline Myoma uteri & $14(46.7 \%)$ \\
\hline $\begin{array}{l}\text { Simple type atypical endometrial } \\
\text { hyperplasia }\end{array}$ & $5(16.7 \%)$ \\
\hline $\begin{array}{l}\text { Simple type atypia-free endometrial } \\
\text { hyperplasia + myoma uteri }\end{array}$ & $7(22.1 \%)$ \\
\hline $\begin{array}{l}\text { Complex type atypia-free endometrial } \\
\text { hyperplasia }\end{array}$ & $3(9.9 \%)$ \\
\hline Serous cystadenoma & $1(3.3 \%)$ \\
\hline \multicolumn{2}{|c|}{$\begin{array}{l}\text { Mean } \pm \text { standard deviation and minimum, maximum values in parenthe- } \\
\text { ses are given. } \\
\text { BMI - body mass index, TAH + BSO - total abdominal hysterectomy and } \\
\text { bilateral salpingoophorectomy, a - number of cases and percentages in } \\
\text { parentheses are given }\end{array}$} \\
\hline
\end{tabular}

sample, Bonferroni correction) of serum mean IMA levels before and after HT ( $3^{\text {rd }}, 6^{\text {th }}$, and $12^{\text {th }}$ months) in the study group are given in Table 2 .

In the study group, in the $3^{\text {rd }}$ month after hormone therapy, there was a statistically significant decrease in the serum IMA level of $0.126 \pm 0.022$ ABSU according to the pre-condition (one way analysis of variance in the associated sample, with Bonferroni correction $p<0.001)$. However, this decrease in serum IMA level disappeared after using HT for 12 months and increased to be close to the state before hormone therapy.

The change in serum mean ischemia-modified albumin (IMA) levels before and after HT in the study group is given in Figure 1.
No side effects were detected in any patient during 12 months of HT.

\section{Discussion}

Cardiovascular diseases constitute the main cause of mortality in women and men. The risk of cardiovascular disease, which is low in women before the menopause, increases rapidly with aging, especially in the period after menopause [7]. The increase in the protective effect of endogenous estrogen with the development of menopause is held responsible for this risk [8].

Hormone therapy is effective in the coagulation and fibrinolytic system. For many years, this effect has been thought to reduce the risk of myocardial infarction and deep vein thrombosis. However, recent studies have shown that, while reducing the risk of myocardial infarction in the future, it increases the risk of venous thromboembolism. It has been determined that young women with early ovarian failure have a lower vascular endothelial function than women with regular menstrual periods of similar age, and have a higher risk of CVD. It succeeded in reversing HT endothelial dysfunction for 6 months in young women with early ovarian failure [9]. However, there have also been studies showing that HT was ineffective or partially effective in correcting endothelial functions $[9,10]$. The endothelium of elderly patients with multiple risk factors was more unresponsive to HT. Endothelial dysfunction with menopause improved with HT in healthy postmenopausal women; however, this effect was not observed in elderly women with multiple cardiovascular risk factors [11]. The negative effect of HT on endothelial functions was directly related to the time since menopause. Estrogen affected endothelial functions more positively in the first 5 years after menopause compared to use more than 5 years later [12].

In the light of observational studies up to the last 10 years, postmenopausal HT was thought to protect the woman from cardiovascular events. Indeed, in many experimental and animal studies, estrogen has been a cardioprotective agent [13-15], and observational studies in women using $\mathrm{HT}$ found that the risk of CAD

Table 2. Changes and statistical analysis of serum mean ischemia modified albumin levels before and after hormone therapy $\left(3^{\text {rd }}, 6^{\text {th }}\right.$, and $12^{\text {th }}$ months) in the study group

\begin{tabular}{lllll}
\hline Groups & & Mean change & $p$ & $95 \% \mathrm{Cl}$ \\
\hline Before HT & $3^{\text {rd }}$ month after HT & $0.126 \pm 0.022$ & $<0.001$ & $0.067-0.185$ \\
\cline { 2 - 5 } & $6^{\text {th }}$ month after HT & $0.064 \pm 0.022$ & 0.025 & 0.999 \\
\cline { 2 - 5 } & $12^{\text {th }}$ month after HT & $0.031 \pm 0.022$ & 0.035 & $-0.028-0.089$ \\
\hline $3^{\text {rd }}$ month after HT & $6^{\text {th }}$ month after HT & $-0.061 \pm 0.022$ & 0.775 & $-0.0225-0.092$ \\
\hline $6^{\text {th }}$ month after HT & $12^{\text {th }}$ month after HT & $-0.033 \pm 0.022$ & 0.002 \\
\hline
\end{tabular}

Mean values \pm standard deviation values are given. One way analysis of variance in the associated sample, with Bonferroni correction test was used. IMA - ischemia modified albumin, HT - hormone therapy 
was reduced by $35-50 \%[16,17]$. However, recent randomized studies have not confirmed the observational study data and raised doubts about HT use by showing that HT does not have clinical benefits in either primary or secondary protection cardiac aspects $[18,19]$.

In a meta-analysis of observational studies conducted until 2000, the beneficial effects of HT on cardiovascular mortality and CAD frequency draw attention [20]. Based on these results, health authorities including the American College of Physicians recommended HT to protect against CAD in postmenopausal women in 1992 [21], and a significant increase in HT use was observed after that date [22]. However, these studies were based on observational data, and when sub-analyses were matched in terms of socioeconomic level and major CAD risk factors, HT lost its protective feature.

When recent prospective studies were examined, a possible relationship was found between postmenopausal obesity and circulating high IMA levels due to oxidative stress [4]. In that study, IMA was measured in the early period. It did not match our current study. The reason for that was the evaluation of long-term results such as 1 year by looking at IMA 4 times as in our study. In another study in 2013 where obese menopausal patients were evaluated, after hormone therapy, IMA levels, CD40 ligand, and platelet levels were evaluated at 3-month and 6-month follow-ups. When the HT study group and control group were compared, it was found that CD40 ligand levels did not decrease, and IMA levels decreased at the end of 6 months. They also stated that platelet levels increased. As a result, they reported that menopause was associated with an increased risk of obesity and associated cardiovascular risk increase, changes in metabolic and endocrine status, and transition to oxidative stress and transition to abdominal fat distribution [5]. In our current study, although early results were consistent in terms of IMA levels, long-term results did not match. The fact that the evaluated cases were patients with obese menopause in the published paper was the main difference from our current study. Another important distinction was that our presented research had long-term IMA data in normal weight surgical menopausal cases.

HERS is the first large-scale randomized clinical trial to examine the relationship between HT and CVD [17]. There was no difference between the groups in terms of non-fatal MI or cardiac death at the end of the fouryear follow-up period. An interesting result of the study was the increase in cardiovascular disease-related risk in the group receiving $\mathrm{HT}$ in the first year following randomization and a decrease in the following years.

The WHI study initiated in 1991 is the most comprehensive prospective randomized study to date, aimed at determining the role of HT in primary cardiovascular protection in healthy postmenopausal women. Similar to the results of the HERS study, it was noteworthy that

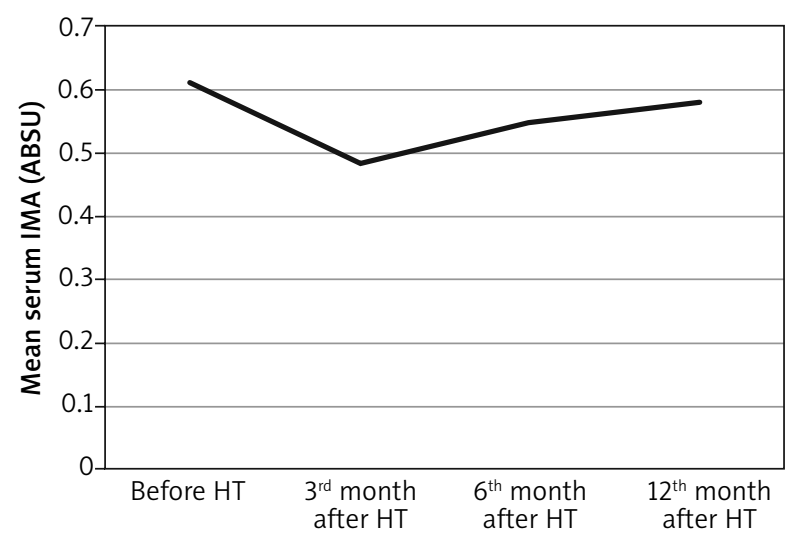

IMA - ischemia modified albumin, ABSU - absorbance units, HT - hormone therapy

Fig. 1. Change of serum mean ischemia modified albumin levels before and after hormone therapy in the study group $\left(3^{\text {rd }}, 6^{\text {th }}\right.$, and $12^{\text {th }}$ months)

increases in $C A D$ risk were observed especially in the first year [1].

In the current study, estrogen therapy was started in patients between the ages of 45 and 55, who did not carry any risk factors for CVD in the postmenopausal period after hysterectomy, and was followed for a year. IMA, an ischemia marker, was used for the risk screening of CVD, and although at the end of the 3rd month IMA levels were low, an increase in IMA levels was observed at the end of 1 year. Results were similar to the values determined at the beginning of HT. These findings are consistent with the WHI and HERS studies; we found that HT does not prevent the ischemic process due to menopause.

According to the results of the study on serum IMA in menopause cases, mean serum IMA level in obese menopause cases was 115 while it was 97 in non-obese menopause cases. Likewise, in menopausal cases with CAD the mean serum IMA level was 117; it was higher than menopausal subjects who were obese or not. This suggests that serum IMA may also be an important marker in menopause cases in terms of showing coronary disease [4].

Starting HT in young, healthy, menopausal patients may restore endothelial dysfunction and may slow down the early stages of development of atherosclerosis. In postmenopausal women aged 45-55 years, we think that HT would be of benefit in the early stages of menopause. However, it should not be recommended to start HT in postmenopausal women for protection against CVD until the results of some ongoing randomized studies are published. It should not be forgotten that there may be many more proven protection methods (such as a healthy diet, regular physical exercise, blood pressure control, diabetes, and dyslipidemia treatment) in this regard.

The strength of the research was that it gave the long-term results of the ischemic process in menopause. 
The low number of cases seemed to be the most important limitation of the study. Another limiting aspect of the research was that only the one-year HT effect was investigated. Planning research including five-year results may reveal the accuracy of our results.

\section{Conclusions}

In this study, increasing IMA levels after one year of HT did not support the argument that HT given during menopause might prevent the ischemic process. The ischemic condition has been in question in menopause. This ischemic process returned in the short term with $\mathrm{HT}$, but long-term treatment did not effectively correct ischemia. This supported the view that HT did not prevent ischemic status, as stated in the WHI study. Our study yielded results for only one year. Further research is needed to generate 5-year results. Nevertheless, according to the results of this study, the message to take home should be as follows: In postmenopausal cases, $\mathrm{HT}$ did not provide any benefit in terms of preventing the ischemic state that caused cardiovascular disease.

\section{Disclosure}

The authors report no conflict of interest.

\section{References}

1. Rossouw JE, Anderson GL, Prentice RL, et al. Risks and benefits of estrogen plus progestin in healthy postmenopausal women: principal results from the women's health initiative randomized controlled trial. Jama 2002; 288: 321-333

2. Paciuc J. Hormone therapy in menopause. Adv Exp Med Biol 2020; 1242: 89-120.

3. Xing D, Nozell S, Chen Y-F, Hage F, Oparil S. Estrogen and mechanisms of vascular protection. Arterioscler Thromb Vasc Biol 2009; 29: 289-295.

4. Kazanis K, Dalamaga M, Kassi E, et al. Serum levels of ischemia modified albumin in overweight/obese postmenopausal women: a potential biomarker of atherosclerotic burden associated with oxidative stress. Maturitas 2011; 70: 182-187

5. Osmanağaoğlu $M$, Karahan $S$, Aran $T$, et al. The effects of hormone therapy on ischemia modified albumin and soluble CD40 ligand levels in obese surgical menopausal women. Clin Exp Obstet Gynecol 2013; 40: 389-392.

6. Bar-Or D, Curtis G, Rao N, Bampos N, Lau E. Characterization of the $\mathrm{Co}^{+}$ and $\mathrm{Ni}^{+}$binding amino-acid residues of the $\mathrm{N}$-terminus of human albumin: an insight into the mechanism of a new assay for myocardial ischemia. Eur J Biochem 2001; 268: 42-48

7. Feng $\mathrm{Y}$, Hong $\mathrm{X}$, Wilker $\mathrm{E}$, et al. Effects of age at menarche, reproductive years, and menopause on metabolic risk factors for cardiovascular diseases. Atherosclerosis 2008; 196: 590-597.

8. Sathyapalan T, Aye M, Rigby AS, et al. Soy isoflavones improve cardiovascular disease risk markers in women during the early menopause. Nutr Metab Cardiovasc Dis 2018; 28: 691-697.

9. Kalantaridou SN, Naka KK, Papanikolaou E, et al. Impaired endothelial function in young women with premature ovarian failure: normalization with hormone therapy. J Clin Endocrinol Metab 2004; 89: 3907-3913.

10. Panazzolo DG, da Silva LH, Maranhao PA, et al. Short-term effects of low-dose estradiol on endothelial function and blood viscosity in nondiabetic postmenopausal overweight women: a double-blind, placebocontrolled study. Menopause 2016; 23: 1114-1121.
11. Ozemek C, Hildreth KL, Blatchford PJ, et al. Effects of resveratrol or estradiol on postexercise endothelial function in estrogen-deficient postmenopausal women. J Appl Physiol 2020; 128: 739-747.

12. Hurtado R, Celani M, Geber S. Effect of short-term estrogen therapy on endothelial function: a double-blinded, randomized, controlled trial. Climacteric 2016; 19: 448-451.

13. Sack MN, Rader D, Cannon RO. Oestrogen and inhibition of oxidation of low-density lipoproteins in postmenopausal women. Lancet 1994; 343: 269-270

14. Purnell JQ, Urbanski HF, Kievit P, Roberts Jr CT, Bethea CL. Estradiol replacement timing and obesogenic diet effects on body composition and metabolism in postmenopausal macaques. Endocrinology 2019; 160: 899-914

15. Savonitto S, Ferri LA, Colombo D. Perimenopause vasomotor symptoms, coronary atherosclerosis and risk of myocardial infarction during menopause: the cardiologist's perspective. Menopause Rev 2018; 17: 53.

16. Mikkola TS. Cardiovascular mortality risk and HRT. Sex Steroids' Effects on Brain, Heart and Vessels, Springer; Berlin 2019, 271-274.

17. Hulley S, Grady D, Bush T, et al. Randomized trial of estrogen plus progestin for secondary prevention of coronary heart disease in postmenopausal women. Jama 1998; 280: 605-613.

18. Cherry N, Gilmour K, Hannaford P, et al. Oestrogen therapy for prevention of reinfarction in postmenopausal women: a randomised placebo controlled trial. Lancet 2002; 360: 2001-2008.

19. Herrington DM, Reboussin DM, Brosnihan KB, et al. Effects of estrogen replacement on the progression of coronary-artery atherosclerosis. N Engl J Med 2000; 343: 522-529.

20. Humphrey LL, Chan BK, Sox HC. Postmenopausal hormone replacement therapy and the primary prevention of cardiovascular disease. Ann Intern Med 2002; 137: 273-284.

21. Grady D, Cummings SR, Petitti D, et al. Guidelines for counseling postmenopausal women about preventive hormone-therapy. Ann Intern Med 1992; 117: 1038-1041.

22. Lobo RA, Pickar JH, Stevenson JC, Mack WJ, Hodis HN. Back to the future: hormone replacement therapy as part of a prevention strategy for women at the onset of menopause. Atherosclerosis 2016; 254: 282-290. 